\title{
Interaction between abomasal blood feeder Haemonchus contortus and intestinal mucosal browser Trichostrongylus colubriformis during co-infection in Boer goats
}

\begin{abstract}
This study was conducted to investigate potential interactions between the abomasal blood feeder Haemonchus contortus and the intestinal mucosal browser Trichostrongylus colubriformis among deliberately infected Boer goats. Faecal and blood samples were collected twice a week for eight weeks from 25 parasite-naïve goats. Correlation analysis and multiple linear regression models were conducted to explore the association between phenotypic variables and variables taken at necropsy. Positive associations were identified between total FEC and $\log \mathrm{T}$. colubriformis number $(\mathrm{r}=0.62, \mathrm{p}<0.05)$ as well as between $\operatorname{IgA}$ and peripheral eosinophil counts $(\mathrm{r}=0.65, \mathrm{p}<0.05)$. A negative correlation was observed between $\mathrm{T}$. colubriformis and $\log \mathrm{H}$. contortus number $(\mathrm{r}=-0.56, \mathrm{p}<0.05)$. Multiple linear regression models show that $\mathrm{H}$. contortus and $\mathrm{T}$. colubriformis interacted with each other. $\mathrm{T}$. colubriformis appeared to contribute more significantly to the variation of FEC than H. contortus. Coinfection induced an IgA response which was only effective against T. colubriformis but not protective against $\mathrm{H}$. contortus infection. This could be seen via significant associations of $\operatorname{IgA}$ with both nematode species but with the effect of IgA differing for $\mathrm{H}$. contortus and $\mathrm{T}$. colubriformis. In this study, H. contortus infection was not detrimental to the goats with no observed impact on PCV. This could be because the growth of T. colubriformis as represented by its length was associated with reduced number and composite burden of $\mathrm{H}$. contortus during co-infection, or possibly due to low infection dosage. Improved understanding of the impact of H. contortus and T. colubriformis and their interaction from natural co-infection studies is beneficial for a better understanding of the goat-parasite interaction and its potential impacts on the health and productivity of animals.
\end{abstract}

Keyword: Haemonchus contortus; Trichostrongylus colubriformis; Goats; Interaction; Faecal; Egg counts; IgA; Eosinophil; Packed cell volume 